\title{
Induction of altered mRNA expression profiles caused by fibrous and granular dust
}

\author{
SIMONE HELMIG $^{1}$, ELKE DOPP $^{2,3}$, SIBYLLE WENZEL $^{1}$, DIRK WALTER $^{1}$ and JOACHIM SCHNEIDER ${ }^{1}$ \\ ${ }^{1}$ Institute and Outpatient Clinic for Occupational and Social Medicine, Justus-Liebig University, D-35392 Giessen; \\ ${ }^{2}$ Institute of Hygiene and Occupational Medicine, University of Duisburg-Essen, D-45122 Essen; \\ ${ }^{3}$ Centre for Water and Environmental Research (ZWU), University of Duisburg-Essen, D-45141 Essen, Germany
}

Received April 17, 2013; Accepted October 2, 2013

DOI: $10.3892 / \mathrm{mmr} .2013 .1765$

\begin{abstract}
Natural and synthetic fibres and particles are being introduced into the workplace and environment daily. Comparative analyses of the induced signalling pathways are essential in order to understand the potential hazards of these particles. To identify the molecular characteristics of particles and fibres, we selected crocidolite and chrysotile asbestos as representatives for fibered dust and titanium dioxide $\left(\mathrm{TiO}_{2}\right)$ (100-200 nm), zirconium dioxide $\left(\mathrm{ZrO}_{2}\right)(50-100 \mathrm{~nm})$ and hematite $\left(\mathrm{Fe}_{2} \mathrm{O}_{3}\right)(20 \mathrm{~nm})$ as representatives for bio-persistent granular dust. SV-40 virus-transformed human bronchial epithelial cells (BEAS-2B) were exposed to well-defined fibres and particles. RT ${ }^{2}$ Profiler $^{\mathrm{TM}}$ PCR Array Human Stress $\&$ Toxicity PathwayFinder was used to compare the relative mRNA expression of 84 genes. A detailed characterization of the dust samples used in this study was accomplished to ensure comparability to other studies. Investigation of mRNA expression of 84 signalling molecules attributed to pathways such as DNA damage and repair; oxidative/metabolic stress; growth arrest and senescence; inflammation, proliferation and carcinogenesis; and heat shock and apoptosis revealed that crocidolite and chrysotile asbestos induced mRNA expression of pathway molecules involved in proliferation and carcinogenesis, as well as inflammation. Titanium dioxide, zirconium dioxide and hematite mainly induced pathway molecules responsible for oxidative/metabolic stress and inflammation. Our findings suggest that the hazards of fibered dust mainly include the induction of direct toxicity by altering signalling pathways such as carcinogenesis and proliferation, while granular dust shows indirect toxicity by altering signalling pathways involved in inflammatory processes. PCR arrays, therefore, may be a helpful tool to estimate the hazard risk of new materials.
\end{abstract}

Correspondence to: Dr Simone Helmig, Institute and Outpatient Clinic for Occupational and Social Medicine, Justus-Liebig University, Aulweg 129, D-35392 Giessen, Germany

E-mail: simone.helmig@arbmed.med.uni-giessen.de

Key words: crocidolite, chrysotile, titanium dioxide, zirconium dioxide, hematite, human stress and toxicity pathway, screening tests

\section{Introduction}

Human populations are exposed to environmental and occupational fibrous and granular dust. The number of synthetic or natural fibres and particles being introduced into the environment is continuously increasing. Due to the increasing number and compositional heterogeneity of potentially harmful fibres and particles, there is a crucial need to understand the mechanisms of their pathogenicity.

Lately, nano-sized particles with a diameter below $100 \mathrm{~nm}$ have become the focus of attention, as they are predicted to have a higher toxic potential as a result of their high surface/mass ratios. However, the crystalline structure, surface properties, solubility and particle size are also known to be relevant parameters (1). Therefore an accurate characterization of the particles is essential to allow an interpretation of the results of a study.

It is reasonable to categorise particles and fibres by their molecular effects. To identify the molecular characteristics of particles and fibres we used Union Internationale Contre le Cancer (UICC) crocidolite and chrysotil asbestos as fibered dust and titanium dioxide $\left(\mathrm{TiO}_{2}\right)$ as well as zirconium dioxide $\left(\mathrm{ZrO}_{2}\right)$ as representatives for bio-persistent granular dust. Hematite $\left(\mathrm{Fe}_{2} \mathrm{O}_{3}\right)$ represents a nano-sized ultrafine dust with an iron $(\mathrm{Fe})$ content of $\sim 70 \%$. Asbestos is known to be a carcinogen associated with the induction of lung cancer, mesothelioma and lung fibrosis (2). DNA damage and apoptosis are important downstream effects of asbestos, which occur in all the major lung target cells studied (3). Exposure to asbestos fibres causes alterations in cell signalling (4) and induction of various pro-inflammatory molecules, such as cytokines $(5,6)$. The pathogenicity of various types of asbestos fibres is thought to be associated with fibre size, geometry and surface composition (7). The iron content in particular has to be considered when assessing the toxicity of asbestos fibres. Crocidolite $\left(\mathrm{Na}_{2}\left[\mathrm{Fe}^{3+}\right]_{2}\left[\mathrm{Fe}^{2+}\right]_{3} \mathrm{Si}_{8} \mathrm{O}_{22}[\mathrm{OH}]_{2}\right)$ typically has a high iron content of $\sim 26 \%$, while the iron content in chrysotile $\left(\mathrm{Mg}_{6} \mathrm{Si}_{4} \mathrm{O}_{10}[\mathrm{OH}]_{8}\right)$ ranges between 1 and $6 \%$, and is primarily present as a surface contaminant (8).

In order to verify that the evoked effects are not only due to the iron content of the investigated particles, we used hematite with an iron content of $\sim 70 \%$. Hematite, the hexagonal modification of iron (III) oxide $\left(\alpha-\mathrm{Fe}_{2} \mathrm{O}_{3}\right)$ is the most important industrial iron oxide used. 
Titanium dioxide, also known as titanium (IV) oxide, is the naturally occurring oxide of titanium, which is commercially used in a wide range of products, such as paint, varnishes, paper coating and cosmetics $(9,10)$. Micro-sized titanium dioxide is suggested to be biologically inert $(11,12)$, although an inflammatory response has been described (10). Particles can generate reactive oxygen species; particularly in the case of nano-sized particles, DNA adducts are observed in human lung cells $(9,13)$. Additionally, increased micronucleus formation and DNA breakage, as well as activation of DNA damage checkpoint kinases in nano- $\mathrm{TiO}_{2}$-treated lymphocytes, have been demonstrated (14).

Zirconium dioxide, also known as zirconia, is used in various products, such as ceramic materials, scratch resistant varnishes and coatings, as well as in medical implants $(15,16)$.

The aim of this study was to compare the effects of well-defined fibres (UICC, crocidolite and chrysotile 'A') and different size particles (titanium dioxide, zirconium dioxide and hematite) on human bronchial epithelial cells (BEAS-2B). We focused on the mRNA expression of 84 signalling molecules attributed to pathways such as 'DNA damage and repair', 'oxidative/metabolic stress', 'growth arrest and senescence', 'inflammation', 'proliferation and carcinogenesis', 'heat shock' and 'apoptosis'.

\section{Materials and methods}

Materials. Crocidolite asbestos (UICC, South African NB \#4173-111-3) and chrysotil asbestos (UICC, Rhodesian NB \#4173-11-2) were used as standard references for bio-persistent fibrous dust. Titanium dioxide anatase (Sigma-Aldrich Chemie GmbH, Steinheim, Germany; AL232033) and zirconium dioxide (Sigma-Aldrich Chemie GmbH; AL230693) represented bio-persistent granular dust. Hematite, $\alpha-\mathrm{Fe}_{2} \mathrm{O}_{3}$ (Nanopowder 544884, Sigma-Aldrich Chemie $\mathrm{GmbH}$ ) was used to represent ultrafine particles.

Characterization of dust materials. For a detailed description of the characterization method, refer to a former paper (17). Scanning electron microscopy (SEM; Hitachi S-2700, Chiyoda, Japan) was used to identify particle geometry as well as the microstructure of the samples. The element analysis resulted from energy dispersive X-ray (EDX). To optimize the conductivity (electron beam), all samples were deposited with a very fine gold $(\mathrm{Au})$ layer using a sputtering technique. Transmission electron microscopy (TEM) analysis combined with electron diffraction (detection of crystallinity) was performed using a transmission electron microscope H-600 (Hitachi, Japan). Thermogravimetry (TG) measurements (corundum crucibles, heating rate $5 \mathrm{~K} / \mathrm{min}$ and synthetic air atmosphere) for controlling impurities such as water were conducted using a thermo balance TG 209 F1 Iris (NETZSCH-Gerätebau GmbH, Selb, Germany).

Culture conditions. SV-40 virus-transformed BEAS-2B cells were obtained from the European collection of cell cultures (ECCC, 95102433). Approximately 10 million cells $\left(10 \times 10^{6}\right)$ after trypsinization and counting using a haemocytometer were plated in $75 \mathrm{~cm}^{2}$ flasks (Falcon; Franklin Lakes, NJ, USA). The cells were grown in $15 \mathrm{ml} \mathrm{Gibco}{ }^{\circledR}$ RPMI 1640

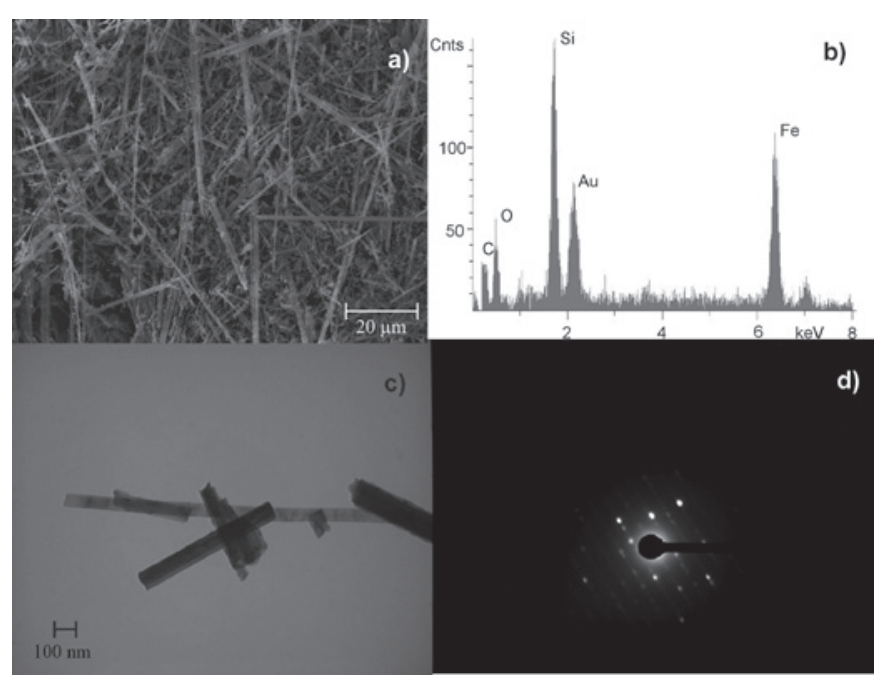

Figure 1. Union Internationale Contre le Cancer (UICC) crocidolite asbestos fibres. (a) Scanning electron microscopy (SEM) (magnification, x1,000) (b) energy dispersive X-ray (EDX)-analysis, (c) transmission electron microscopy (TEM) (magnification, $x 40,000)$ and (d) electron diffraction pattern.

media containing $10-15 \%$ fetal calf serum (FCS), $0.5 \%$ gentamycin, $1 \%$ L-glutamine and $1 \%$ amphotericin. The cultures were maintained at $37^{\circ} \mathrm{C}$ and $5 \% \mathrm{CO}_{2}$. After a 24-h pre-incubation, the cells were exposed to crocidolite $(5 \mu \mathrm{g} /$ $\left.\mathrm{cm}^{2}\right)$, chrysotil $\left(1 \mu \mathrm{g} / \mathrm{cm}^{2}\right)$, zirconium dioxide $\left(10 \mu \mathrm{g} / \mathrm{cm}^{2}\right)$, titanium dioxide $\left(10 \mu \mathrm{g} / \mathrm{cm}^{2}\right)$ or hematite $\left(10 \mu \mathrm{g} / \mathrm{cm}^{2}\right)$ for $48 \mathrm{~h}$. Unexposed cells served as negative controls. All experiments were repeated 4 times. Cytotoxicity and genotoxicity analyses were investigated intensively in various cell systems for crocidolite and chrysotile $(18-21)$, titanium dioxide $(22,23)$ and hematite $(22,24)$. Based on the results of the above study, the particle concentrations did not show any loss of viability in the BEAS-2B cell line. Additionally, the same concentrations and incubation times were used in numerous published studies and therefore the results are comparable.

$m R N A$ extraction and reverse transcription. After washing twice with $\mathrm{PBS}\left(37^{\circ} \mathrm{C}\right)$, cells were trypsinized for $\sim 30 \mathrm{sec}$ with $10 \mathrm{ml}$ of $0.05 \%$ trypsin and incubated for $10 \mathrm{~min}$ in $37^{\circ} \mathrm{C}$. Detached cells then were resuspended in $5 \mathrm{ml}$ ice-cold PBS and centrifuged at $400 \mathrm{xg}$ (without brakes) for $10 \mathrm{~min}$ in $15-\mathrm{ml}$ centrifuge tubes. This step was repeated with $1 \mathrm{ml}$ of ice-cold PBS in $1.5 \mathrm{ml}$ Eppendorf tubes. mRNA was extracted immediately with RNeasy Mini kit ${ }^{\circledR}$ (Qiagen, Hilden, Germany) in accordance with the manufacturer's instructions. Reverse transcription was accomplished with the RT ${ }^{2}$ First Strand kit (Qiagen) as suggested by the manufacturer.

RT $T^{2}$ Profiler PCR Arrays ${ }^{\circledR}$. The RT ${ }^{2}$ RNA QC PCR Array ${ }^{\circledR}$ (SaBiosciences, Qiagen) was used to test for RNA quality and inhibitors of RT-PCR analyses. For quantitative comparison of mRNA levels, real-time PCR was performed using $\mathrm{RT}^{2}$ Profiler PCR Arrays ${ }^{\circledR}$ Human Stress \& Toxicity PathwayFinder PCR Array ${ }^{\circledR}$ (SaBiosciences). For each condition, four assays were carried out as independent samples. Gene expression was related to the mean expression of $\beta_{2}$ microglobulin (B2M) and hypoxanthine phosphoribosyltransferase 1 (HPRT) as house- 


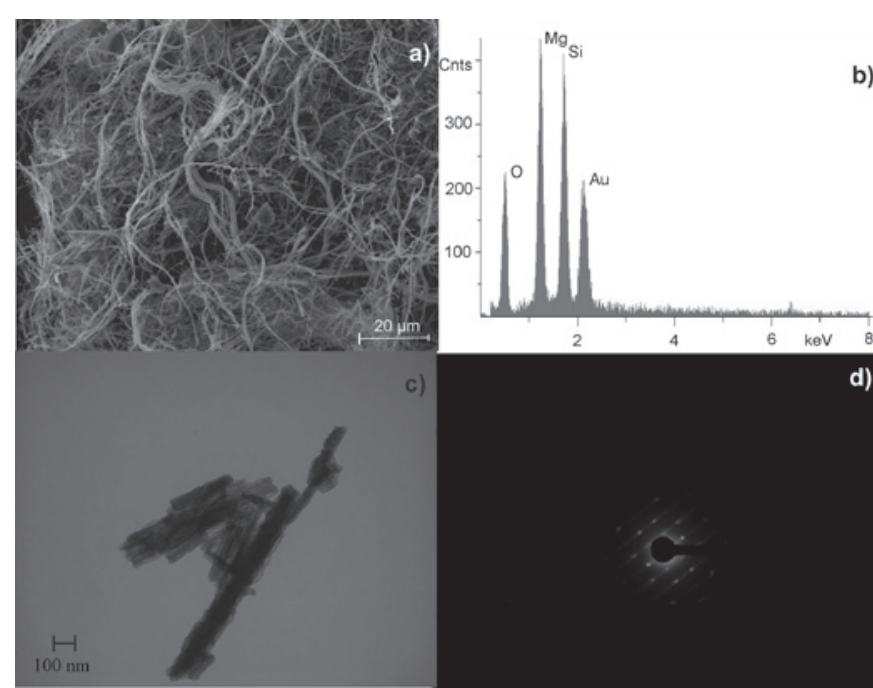

Figure 2. Union Internationale Contre le Cancer (UICC) chrysotile asbestos fibres. (a) Scanning electron microscopy (SEM) (magnification, x1,000), (b) energy dispersive X-ray (EDX) analysis, (c) transmission electron microscopy (TEM) (magnification, x40,000) and (d) electron diffraction pattern.

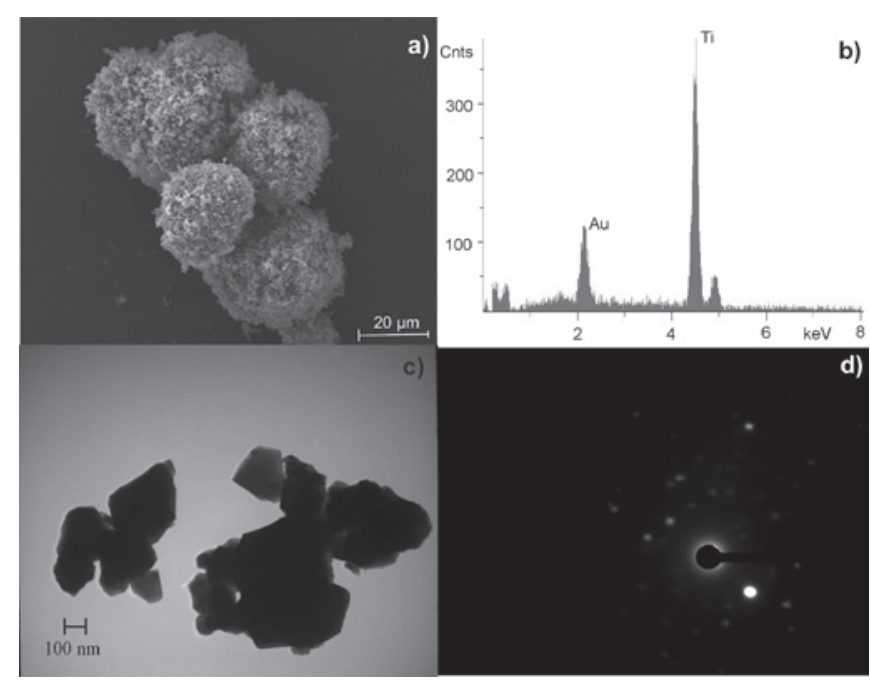

Figure 3. Irregularly shaped crystalline titanium dioxide $\left(\mathrm{TiO}_{2}\right)$ aggregates (a) Scanning electron microscopy (SEM) (magnification, x1,000), (b) energy dispersive X-ray (EDX) analysis, (d) transmission electron microscopy (TEM) (magnification, x40,000) and (d) electron diffraction pattern.

keeping genes, since these were the two most stable of the five housekeeping genes included in the array. Only $\mathrm{Ct}$ values $<35$ were included in the calculations.

Statistical analysis. Calculations of expression were performed with the $2^{-\Delta \Delta C T}$ method according to Pfaffl (25). For analysis the PCR Array Data Analysis Software (Excel \& Web-based) provided by SaBiosciences was used. The cut-off was set to $\mathrm{CT}>35$. The P-values are calculated based on a Student's t-test of the replicate $2^{-\Delta \mathrm{Ct}}$ values for each gene in the control and treatment groups. Results are shown as the mean of four samples for each condition in relation to the mean of four control samples. All statistical analyses were performed using the statistical software package, SPSS, 17.0 (SPSS, Inc.,

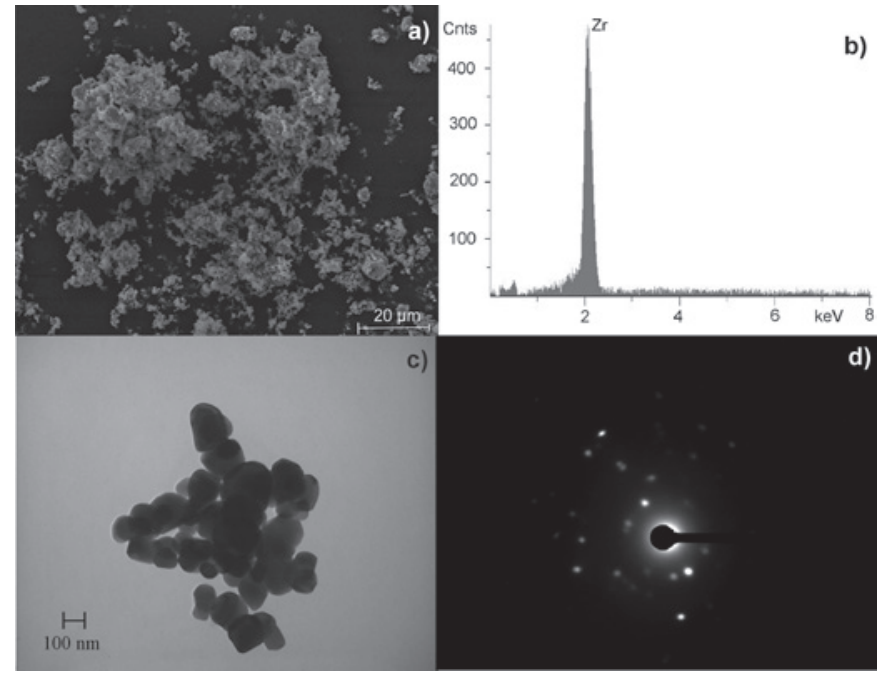

Figure 4. Crystalline zirconium dioxide $\left(\mathrm{ZrO}_{2}\right)$ aggregates. (a) Scanning electron microscopy (SEM) (magnification, x1,000), (b) energy dispersive X-ray (EDX)-analysis, (c) transmission electron microscopy (TEM) (magnification, $\mathrm{x} 40,000)$ and (d) electron diffraction pattern.

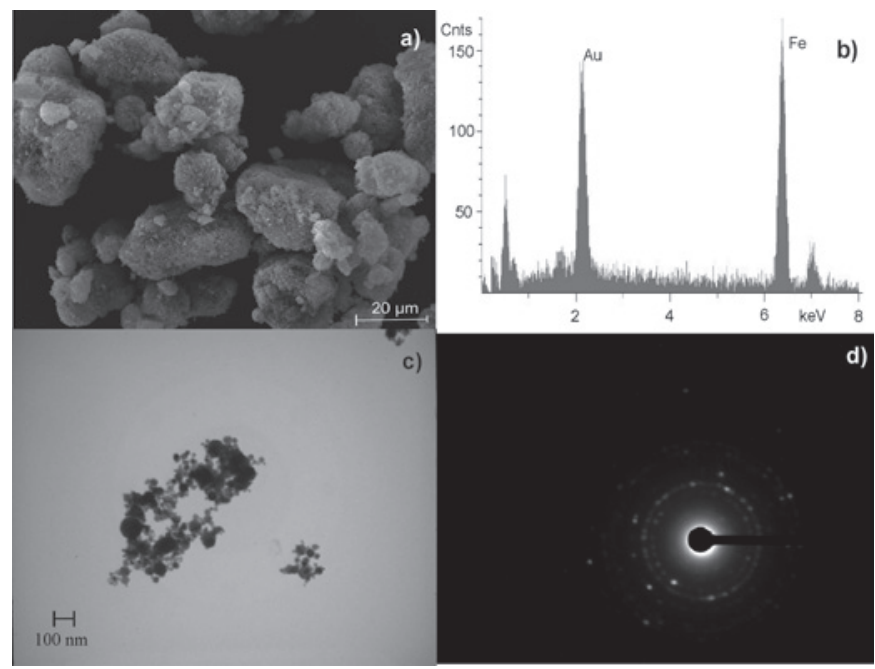

Figure 5. Agglomerates and aggregates of crystalline nano-sized hematite. (a) Scanning electron microscopy (SEM) (magnification, x1,000), (b) energy dispersive X-ray (EDX) analysis, (c) transmission electron microscopy (TEM) (magnification, $\mathrm{x} 40,000)$ and (d) electron diffraction pattern.

Chicago, IL, USA). P<0.05 was considered to indicate a statistically significant difference.

\section{Results}

Characterization of dust samples. UICC crocidolite South African $\left(\mathrm{Na}_{2}\left(\mathrm{Fe}_{3}{ }^{2+} \mathrm{Fe}_{2}{ }^{3+}\left[(\mathrm{OH})_{2} \mid \mathrm{Si}_{8} \mathrm{O}_{22}\right]\right)\right.$ was shown to have $3,800 \mathrm{fibres} / \mathrm{ml}$ at a length of $>5 \mu \mathrm{m}$ and a diameter of $<3 \mu \mathrm{m}$. The length to diameter ratio was at least 3:1 (WHO fibres). Crocidolite is a rigid and rod-like fibre with characteristic iron content (Fig. 1). Gold (Au) was detected in all EDX analyses due to the sputtering preparation technique.

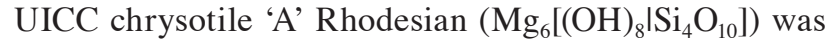
shown to have 200 fibres $/ \mathrm{ml}$ at a length of $>5 \mu \mathrm{m}$ and a diameter of $<3 \mu \mathrm{m}$. The length to diameter ratio was at least 


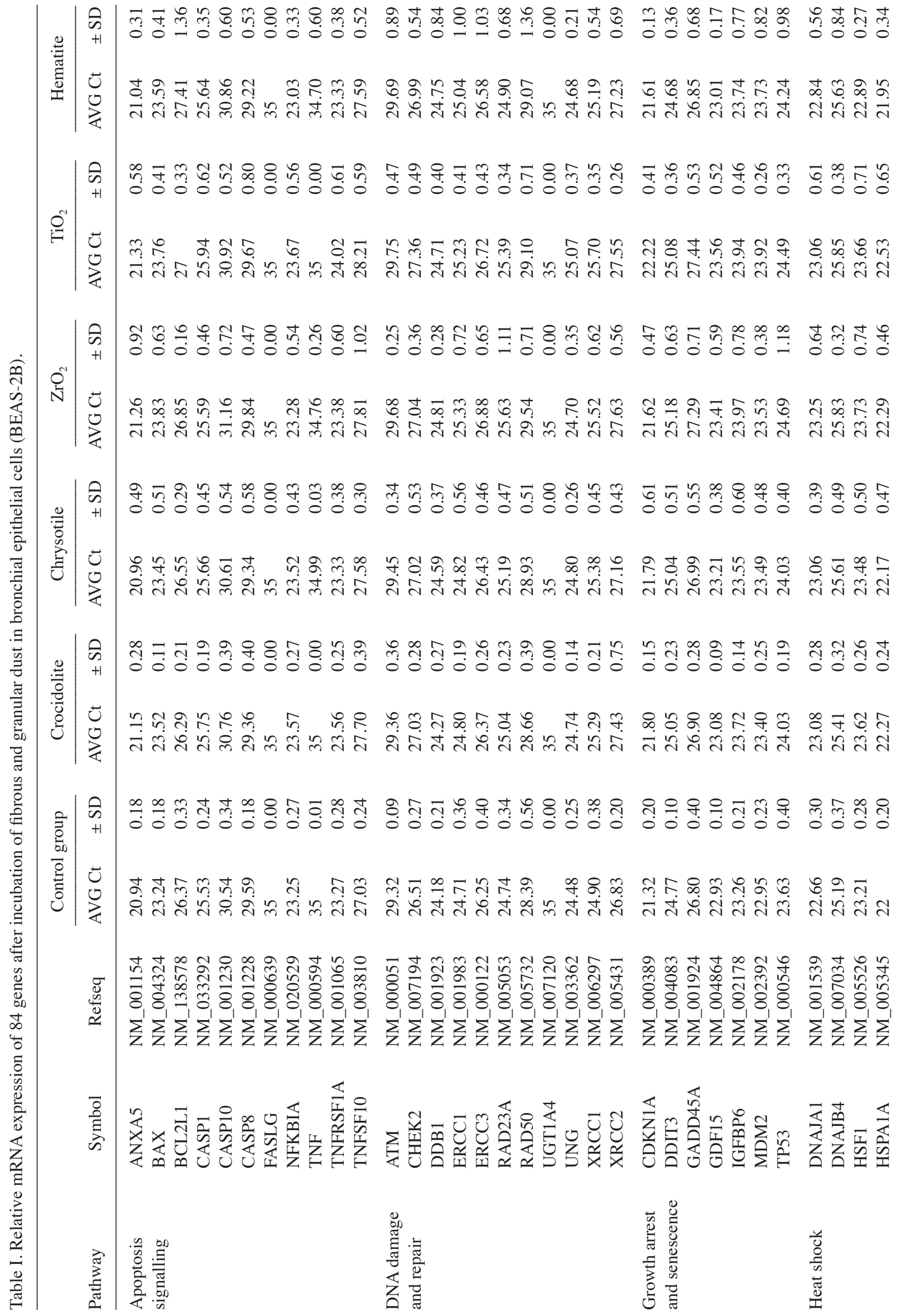




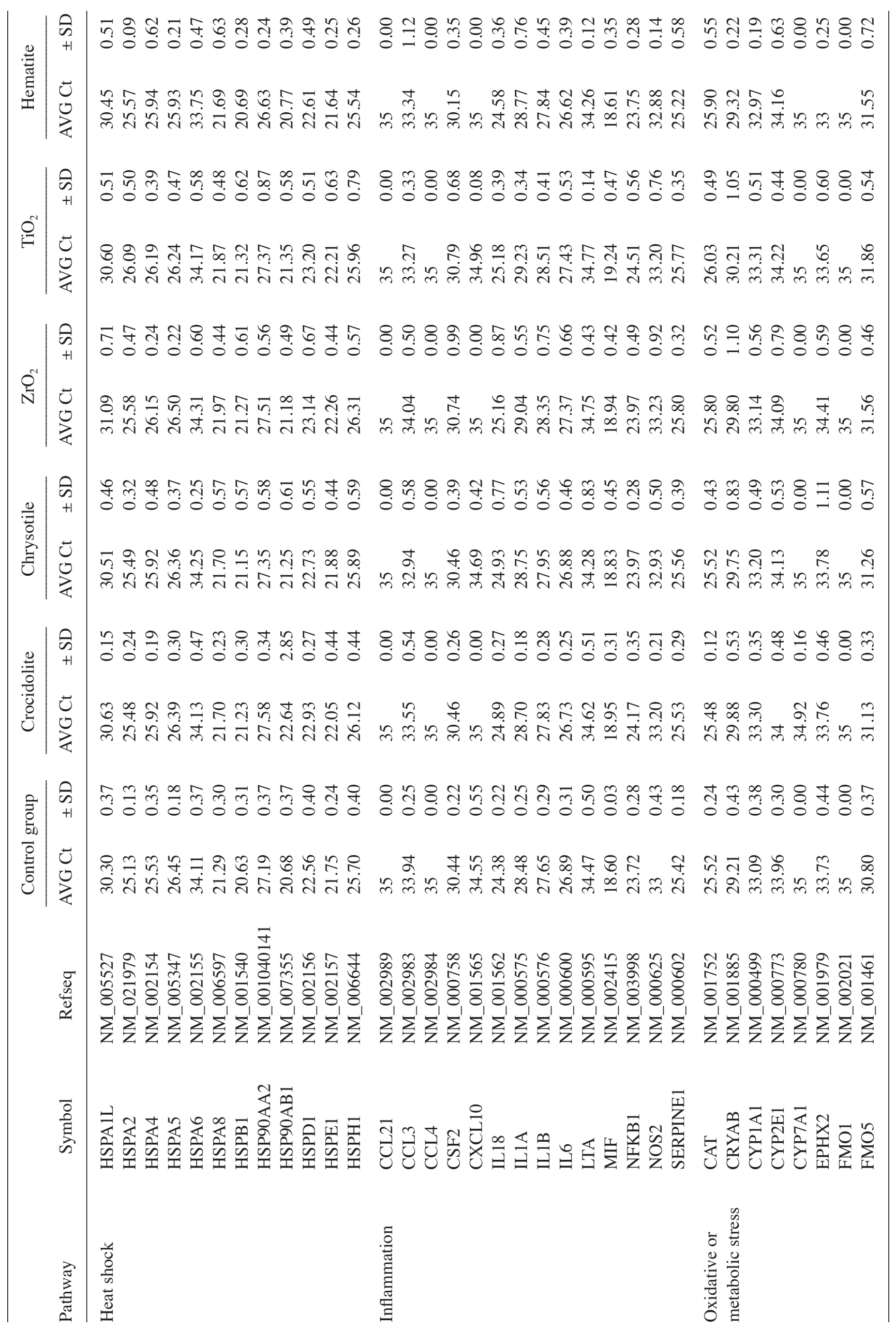




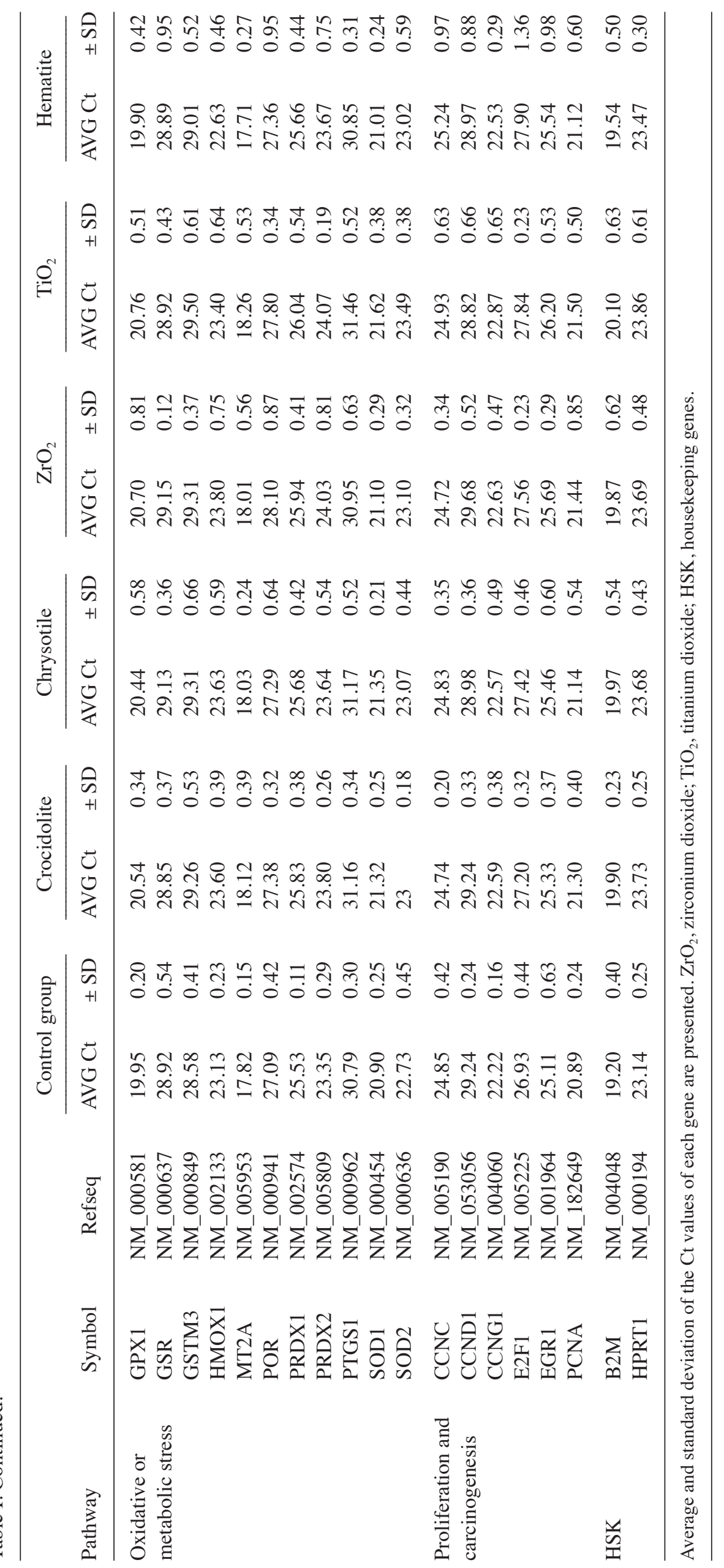


Table II. Comparing mRNA expression $(95 \% \mathrm{CI})$ of DNA damage and repair molecules.

\begin{tabular}{|c|c|c|c|c|c|}
\hline $\begin{array}{l}\text { DNA damage } \\
\text { and repair }\end{array}$ & $\begin{array}{l}\text { Crocidolite } \\
\text { fold change } \\
(95 \% \mathrm{CI})\end{array}$ & $\begin{array}{l}\text { Chrysotile } \\
\text { fold change } \\
(95 \% \mathrm{CI})\end{array}$ & $\begin{array}{l}\text { Titanium dioxide } \\
\text { fold change } \\
(95 \% \mathrm{CI})\end{array}$ & $\begin{array}{l}\text { Zirconium dioxide } \\
\text { fold change } \\
(95 \% \mathrm{CI})\end{array}$ & $\begin{array}{l}\text { Hematite } \\
\text { fold change } \\
(95 \% \mathrm{CI})\end{array}$ \\
\hline ATM & $1.53^{\mathrm{a}}(1.07-1.98)$ & - & - & - & - \\
\hline CHEK2 & - & - & - & - & - \\
\hline DDB1 & $1.48^{\mathrm{a}}(1.19-1.76)$ & - & - & - & - \\
\hline ERCC1 & $1.47^{\mathrm{a}}(1.18-1.76)$ & $1.46^{\mathrm{a}}(1.10-1.83)$ & - & - & - \\
\hline ERCC3 & $1.44^{\mathrm{a}}(1.21-1.68)$ & $1.39^{\mathrm{a}}(1.14-1.64)$ & - & - & - \\
\hline RAD23A & $1.27^{\mathrm{a}}(1.06-1.48)$ & - & - & - & - \\
\hline RAD50 & $1.29^{\mathrm{b}}(1.03-1.57)$ & - & - & $-1.45^{\mathrm{c}}[-1.19-(-1.85)]$ & - \\
\hline UGT1A4 & - & - & - & - & - \\
\hline UNG & $1.31^{\mathrm{a}}(1.13-1.49)$ & - & - & $1.31^{\mathrm{a}}(1.04-1.58)$ & - \\
\hline $\mathrm{XRCC} 1$ & $1.20^{\mathrm{a}}(1.07-1.34)$ & - & - & - & - \\
\hline XRCC2 & - & $1.26^{\mathrm{a}}(1.04-1.47)$ & - & - & - \\
\hline
\end{tabular}

${ }^{\mathrm{a}} \mathrm{P}<0.050,{ }^{\mathrm{b}} \mathrm{P}<0.055$ and ${ }^{\mathrm{c}} \mathrm{P}=0.038$. CI, confidence interval.

Table III. Comparing mRNA expression (95\% CI) of oxidative and metabolic stress molecules.

\begin{tabular}{|c|c|c|c|c|c|}
\hline $\begin{array}{l}\text { Oxidative or } \\
\text { metabolic stress }\end{array}$ & $\begin{array}{l}\text { Crocidolite } \\
\text { fold change } \\
(95 \% \mathrm{CI})\end{array}$ & $\begin{array}{l}\text { Chrysotile } \\
\text { fold change } \\
(95 \% \mathrm{CI})\end{array}$ & $\begin{array}{l}\text { Titanium dioxide } \\
\text { fold change } \\
(95 \% \mathrm{CI})\end{array}$ & $\begin{array}{l}\text { Zirconium dioxide } \\
\text { fold change } \\
(95 \% \mathrm{CI})\end{array}$ & $\begin{array}{l}\text { Hematite } \\
\text { fold change } \\
(95 \% \mathrm{CI})\end{array}$ \\
\hline CAT & $1.62^{\mathrm{a}}(1.25-1.99)$ & $1.58^{\mathrm{a}}(1.15-2.01)$ & - & - & - \\
\hline CRYAB & - & - & - & - & - \\
\hline CYP1A1 & $1.35^{\mathrm{a}}(1.07-1.63)$ & - & - & $1.47^{\mathrm{a}}(1.18-1.76)$ & $1.37^{\mathrm{b}}(1.01-1.72)$ \\
\hline $\mathrm{CY}$ & - & - & - & - & - \\
\hline CYP7A1 & - & - & - & - & - \\
\hline EPHX2 & $1.53^{\mathrm{a}}(1.01-1.72)$ & & - & - & $2.09^{\mathrm{a}}(1.12-3.05)$ \\
\hline FMO1 & - & - & - & - & - \\
\hline FMO5 & - & - & - & - & - \\
\hline GPX1 & - & - & - & - & - \\
\hline GSR & $1.64^{\mathrm{a}}(1.11-2.18)$ & $1.37^{\mathrm{a}}(1.04-1.69)$ & $1.76^{\mathrm{a}}(1.02-2.50)$ & - & - \\
\hline GSTM3 & - & - & - & - & - \\
\hline HMOX1 & - & - & - & $1.46^{\mathrm{a}}(1.19-1.73)$ & $1.79^{a}(1.56-2.01)$ \\
\hline MT2A & $1.28^{\mathrm{b}}(1.02-1.54)$ & - & $1.30^{\mathrm{a}}(1.09-1.50)$ & $1.34^{\mathrm{a}}(1.15-1.53)$ & $1.37^{\mathrm{a}}(1.12-1.61)$ \\
\hline POR & $1.28^{\mathrm{a}}(1.13-1.43)$ & - & - & - & - \\
\hline PRDX1 & $1.28^{\mathrm{b}}(1.01-1.55)$ & $1.42^{\mathrm{a}}(1.09-1.76)$ & $1.23^{\mathrm{b}}(1.01-1.46)$ & - & - \\
\hline PRDX2 & & $1.29^{\mathrm{a}}(1.03-1.54)$ & - & - & - \\
\hline PTGS1 & - & - & - & $1.37^{\mathrm{a}}(1.16-1.58)$ & $1.21^{\mathrm{a}}(1.04-1.39)$ \\
\hline SOD1 & - & - & - & - & - \\
\hline SOD2 & - & - & - & - & - \\
\hline
\end{tabular}

${ }^{\mathrm{a}} \mathrm{P}<0.050,{ }^{\mathrm{b}} \mathrm{P}<0.056 . \mathrm{CI}$, confidence interval.

3:1 (WHO fibres). Chrysotile has a curly, pliable structure with nearly equal magnesium $(\mathrm{Mg}) /$ silicon $(\mathrm{Si})$ distribution (Fig. 2).

Irregularly shaped crystalline titanium dioxide aggregates (diameter, 1-3 $\mu \mathrm{m}$ ) were observed (Fig 3). The micro-sized aggregates were composed of $\sim 20$ primary particles with a diameter between 100 and $200 \mathrm{~nm}$. The specific surface (BET) of titanium dioxide was $9.9 \mathrm{~m}^{2} / \mathrm{g}$. Evaluation of the BET (for titanium dioxide and zirconium dioxide) was performed by K.-P- Company for surface- and solid state analysis $\mathrm{mbH}$ (o.f.u), Hamburg, Germany (Report B0104014 for the Federal Institute of Occupational Safety and Medicine, May 2001).

For zirconium dioxide, an aggregate diameter of 1-2 $\mu \mathrm{m}$ was determined. The crystalline aggregates were composed 
Table IV. Comparing mRNA expression $(95 \% \mathrm{CI})$ of DNA growth arrest and senescence molecules.

\begin{tabular}{|c|c|c|c|c|c|}
\hline $\begin{array}{l}\text { Growth arrest } \\
\text { and senescence }\end{array}$ & $\begin{array}{l}\text { Crocidolite } \\
\text { fold change } \\
(95 \% \mathrm{CI})\end{array}$ & $\begin{array}{l}\text { Chrysotile } \\
\text { fold change } \\
(95 \% \mathrm{CI})\end{array}$ & $\begin{array}{l}\text { Titanium dioxide } \\
\text { fold change } \\
(95 \% \mathrm{CI})\end{array}$ & $\begin{array}{l}\text { Zirconium dioxide } \\
\text { fold change } \\
(95 \% \mathrm{CI})\end{array}$ & $\begin{array}{l}\text { Hematite } \\
\text { fold change } \\
(95 \% \mathrm{CI})\end{array}$ \\
\hline \multicolumn{6}{|l|}{ CDKN1A } \\
\hline DDIT3 & $1.30^{\mathrm{a}}(1.02-1.57)$ & $1.30^{\mathrm{a}}(1.02-1.57)$ & $1.40^{\mathrm{a}}(1.02-1.57)$ & - & - \\
\hline GADD45A & $1.46^{\mathrm{a}}(1.11-1.80)$ & - & - & - & - \\
\hline GDF15 & $1.41^{\mathrm{a}}(1.13-1.69)$ & - & - & - & - \\
\hline IGFBP6 & - & $1.30^{\mathrm{a}}(1.13-1.69)$ & - & - & - \\
\hline MDM2 & - & - & - & - & - \\
\hline TP53 & $1.19^{\mathrm{a}}(1.02-1.35)$ & - & - & - & - \\
\hline
\end{tabular}

${ }^{\mathrm{a}} \mathrm{P}<0.050$. CI, confidence interval.

Table V. Comparing mRNA expression $(95 \% \mathrm{CI})$ of inflammatory molecules.

\begin{tabular}{|c|c|c|c|c|c|}
\hline Inflammation & $\begin{array}{l}\text { Crocidolite } \\
\text { fold change } \\
(95 \% \mathrm{CI})\end{array}$ & $\begin{array}{l}\text { Chrysotile } \\
\text { fold change } \\
(95 \% \mathrm{CI})\end{array}$ & $\begin{array}{l}\text { Titanium dioxide } \\
\text { fold change } \\
(95 \% \mathrm{CI})\end{array}$ & $\begin{array}{l}\text { Zirconium dioxide } \\
\text { fold change } \\
(95 \% \mathrm{CI})\end{array}$ & $\begin{array}{l}\text { Hematite } \\
\text { fold change } \\
(95 \% \mathrm{CI})\end{array}$ \\
\hline \multicolumn{6}{|l|}{ CCL21 } \\
\hline CCL3 & $2.05^{\mathrm{a}}(1.34-2.77)$ & $3.16^{\mathrm{a}}(1.47-4.85)$ & $2.79^{a}(1.63-3.94)$ & - & - \\
\hline CCL4 & - & - & - & - & - \\
\hline CSF2 & $1.54^{\mathrm{a}}(1.28-1.80)$ & - & - & - & $1.54^{\mathrm{a}}(1.20-1.88)$ \\
\hline CXCL10 & - & - & - & - & - \\
\hline IL18 & - & - & - & - & - \\
\hline IL1A & $1.34^{\mathrm{a}}(1.09-1.59)$ & - & - & - & - \\
\hline IL1B & $1.39^{\mathrm{a}}(1.30-1.47)$ & - & - & - & - \\
\hline IL6 & $1.76^{\mathrm{a}}(1.48-2.04)$ & $1.59^{\mathrm{a}}(1.24-1.95)$ & - & - & $1.53^{\mathrm{a}}(1.34-1.72)$ \\
\hline LTA & - & - & - & - & - \\
\hline MIF & - & $1.35^{\mathrm{a}}(1.34-1.72)$ & - & - & - \\
\hline NFKB1 & - & - & - & - & - \\
\hline NOS2 & - & - & - & - & - \\
\hline SERPINE1 & $1.45^{\mathrm{a}}(1.25-1.65)$ & $1.42^{\mathrm{a}}(1.14-1.70)$ & - & - & $1.45^{\mathrm{a}}(1.14-1.70)$ \\
\hline
\end{tabular}

${ }^{\mathrm{a}} \mathrm{P}<0.050$. CI, confidence interval.

of $\sim 50$ primary particles with a diameter of $\sim 100 \mathrm{~nm}$ (Fig. 4). The specific surface (BET) of zirconium dioxide was $5.9 \mathrm{~m}^{2} / \mathrm{g}$.

Hematite was found to be a spherical formed, nano-sized material. Agglomerates of 0.2-2 $\mu \mathrm{m}$ were formed by 50-500 primary particles with a diameter of $\sim 20 \mathrm{~nm}$. Additionally, smaller aggregates $(<100 \mathrm{~nm})$ were detected by electron microscopy (Fig. 5). The usually observed integration of water within the crystal lattice, caused by the production (precipitation) process of hematite, was excluded by TG (26-28).

After a 48-h exposure to the described fibres and particles, relative mRNA expression of 84 genes were determined four times. The average and standard deviation of the $\mathrm{Ct}$ values of each gene are shown in Table I.

Direct genotoxicity. Molecules of the 'DNA damage and repair' pathway were assigned to direct genotoxicity. In
BEAS-2B cells, UGT1A4 mRNA expression could not be detected. mRNA expression of the 'DNA damage and repair' pathway was induced mainly by crocidolite, followed by chrysotile. Zirconium dioxide significantly upregulated UNG (1.31; $\mathrm{P}=0.043)$ but downregulated RAD50 (-1.45; $\mathrm{P}=0.038)$, while neither titanium dioxide nor hematite altered mRNA expression of 'DNA damage and repair' pathway signalling molecules (Table II).

Indirect genotoxicity. Molecules of the 'oxidative or metabolic stress', 'growth arrest and senescence' as well as 'inflammation' pathway were assigned to indirect genotoxicity. In BEAS-2B cells, Cyp7A1, FMO1, CCL21, CCL4, CXCL10 and LTA mRNA expression could not be detected. The majority of changes in the signalling molecule mRNA expression of the 'oxidative or metabolic stress' pathway were due to crocido- 
Table VI. Comparing mRNA expression (95\% CI) of DNA proliferation and carcinogenesis molecules.

\begin{tabular}{|c|c|c|c|c|c|}
\hline $\begin{array}{l}\text { Proliferation and } \\
\text { carcinogenesis }\end{array}$ & $\begin{array}{l}\text { Crocidolite } \\
\text { fold change } \\
(95 \% \mathrm{CI})\end{array}$ & $\begin{array}{l}\text { Chrysotile } \\
\text { fold change } \\
(95 \% \mathrm{CI})\end{array}$ & $\begin{array}{l}\text { Titanium dioxide } \\
\text { fold change } \\
(95 \% \mathrm{CI})\end{array}$ & $\begin{array}{l}\text { Zirconium dioxide } \\
\text { fold change } \\
(95 \% \mathrm{CI})\end{array}$ & $\begin{array}{l}\text { Hematite } \\
\text { fold change } \\
(95 \% \mathrm{CI})\end{array}$ \\
\hline \multicolumn{6}{|l|}{$\mathrm{CCNC}$} \\
\hline CCND1 & $1.57^{\mathrm{a}}(1.28-1.87)$ & $1.89^{\mathrm{a}}(1.33-2.44)$ & $2.36^{\mathrm{a}}(1.33-2.44)$ & - & - \\
\hline CCNG1 & - & - & - & - & - \\
\hline E2F1 & - & - & - & - & - \\
\hline EGR1 & - & - & - & - & - \\
\hline PCNA & - & - & - & - & - \\
\hline
\end{tabular}

${ }^{\mathrm{a}} \mathrm{P}<0.050$. CI, confidence interval.

Table VII. Comparing mRNA expression (95\% CI) of heat shock molecules.

\begin{tabular}{|c|c|c|c|c|c|}
\hline Heat shock & $\begin{array}{l}\text { Crocidolite } \\
\text { fold change } \\
(95 \% \mathrm{CI})\end{array}$ & $\begin{array}{l}\text { Chrysotile } \\
\text { fold change } \\
(95 \% \mathrm{CI})\end{array}$ & $\begin{array}{l}\text { Titanium dioxide } \\
\text { fold change } \\
(95 \% \mathrm{CI})\end{array}$ & $\begin{array}{l}\text { Zirconium dioxide } \\
\text { fold change } \\
(95 \% \mathrm{CI})\end{array}$ & $\begin{array}{l}\text { Hematite } \\
\text { fold change } \\
(95 \% \mathrm{CI})\end{array}$ \\
\hline DNAJA1 & $1.17^{\mathrm{a}}(1.03-1.31)$ & - & - & - & - \\
\hline DNAJB4 & - & - & - & - & - \\
\hline HSF1 & - & $1.31^{\mathrm{a}}(1.05-1.56)$ & $1.29^{\mathrm{a}}(1.14-1.44)$ & - & $1.57^{\mathrm{a}}(1.33-1.81)$ \\
\hline HSPA1A & $1.30^{\mathrm{a}}(1.10-1.51)$ & - & $1.22^{\mathrm{a}}(1.05-1.38)$ & $1.25^{\mathrm{a}}(1.06-1.44)$ & - \\
\hline HSPA1L & $1.25^{\mathrm{a}}(1.05-1.46)$ & $1.36^{\mathrm{a}}(1.13-1.60)$ & $1.43^{\mathrm{a}}(1.21-1.64)$ & - & - \\
\hline HSPA2 & - & - & - & - & - \\
\hline HSPA4 & - & - & - & - & - \\
\hline HSPA5 & $1.63^{\mathrm{a}}(1.21-2.04)$ & $1.67^{\mathrm{a}}(1.07-2.27)$ & $2.02^{\mathrm{a}}(1.37-2.67)$ & - & $1.81^{\mathrm{a}}(1.25-2.36)$ \\
\hline HSPA6 & - & - & $1.68^{\mathrm{b}}(0.83-2.53)$ & - & - \\
\hline HSPA8 & $1.19^{\mathrm{a}}(1.09-1.28)$ & $1.19^{\mathrm{b}}(1.02-1.35)$ & - & - & - \\
\hline HSPB1 & - & - & - & - & $1.22^{\mathrm{a}}(1.09-1.34)$ \\
\hline HSP90AA2 & $1.20^{\mathrm{a}}(1.03-1.37)$ & - & $1.54^{\mathrm{a}}(1.10-1.98)$ & - & $1.86^{\mathrm{a}}(1.44,2.28)$ \\
\hline HSP90AB1 & - & - & - & - & $1.19^{\mathrm{a}}(1.05-1.32)$ \\
\hline HSPD1 & - & $1.40^{\mathrm{a}}(1.18-1.62)$ & - & - & $1.23^{\mathrm{a}}(1.03-1.43)$ \\
\hline HSPE1 & - & - & - & - & $1.36^{\mathrm{a}}(1.02-1.69)$ \\
\hline HSPH1 & - & - & $1.47^{\mathrm{a}}(1.12-1.82)$ & - & $1.41^{\mathrm{a}}(1.15-1.68)$ \\
\hline
\end{tabular}

${ }^{\mathrm{a}} \mathrm{P}<0.050,{ }^{\mathrm{b}} \mathrm{P}<0.058 . \mathrm{CI}$, confidence interval.

lite. Notably, changes in signalling molecule expression were comparable for chrysotile, zirconium dioxide, titanium dioxide and hematite (Table III).

Both fibres showed a moderate increase in signalling molecule expression of the 'growth arrest and senescence' pathway, while titanium dioxide only induced DDIT3 (1.4, $\mathrm{P}=0.048)$. There was no significant change in mRNA expression due to zirconium dioxide and hematite (Table IV).

Molecules belonging to the 'inflammation' pathway were induced mainly by crocidolite. Chrysotile and hematite provoked a comparable moderate increase in gene expression. Titanium dioxide distinctly induced CCL3 (2.79, P=0.007), while there were no expression changes due to zirconium dioxide (Table V).
Initiation and promotion of carcinogenesis. Molecules of the 'proliferation and carcinogenesis' pathway were assigned to initiation and promotion of carcinogenesis. The only gene of this pathway, which was induced was Cyclin D1 (CCND1). Cyclin D expression was induced by crocidolite (1.57, $\mathrm{P}=0.004)$, chrysotile $(1.89, \mathrm{P}=0.019)$ and titanium dioxide (2.36, $\mathrm{P}=0.007$ ) (Table VI).

Acute toxicity and/or genotoxicity. Molecules of the 'heat shock' and 'apoptosis' pathways were assigned to acute toxicity and/or genotoxicity. Of all investigated pathways, the greatest changes were found within these two pathways. Crocidolite, titanium dioxide and hematite provoked the most changes in mRNA expression of signalling molecules of the 
Table VIII. Comparing mRNA expression (95\% CI) of apoptosis molecules.

\begin{tabular}{|c|c|c|c|c|c|}
\hline Apoptosis & $\begin{array}{l}\text { Crocidolite } \\
\text { fold change } \\
(95 \% \mathrm{CI})\end{array}$ & $\begin{array}{l}\text { Chrysotile } \\
\text { fold change } \\
(95 \% \mathrm{CI})\end{array}$ & $\begin{array}{l}\text { Titanium dioxide } \\
\text { fold change } \\
(95 \% \mathrm{CI})\end{array}$ & $\begin{array}{l}\text { Zirconium dioxide } \\
\text { fold change } \\
(95 \% \mathrm{CI})\end{array}$ & $\begin{array}{l}\text { Hematite } \\
\text { fold change } \\
(95 \% \mathrm{CI})\end{array}$ \\
\hline ANXA5 & - & $1.55^{\mathrm{b}}(1.01-2.08)$ & - & - & - \\
\hline BAX & $1.29^{\mathrm{a}}(1.05-1.53)$ & $1.36^{\mathrm{b}}(1.00-1.72)$ & - & - & - \\
\hline BCL2L1 & $1.66^{\mathrm{a}}(1.30-2.03)$ & - & - & - & - \\
\hline CASP1 & $1.35^{\mathrm{b}}(1.11-1.58)$ & $1.44^{\mathrm{a}}(1.07-1.81)$ & $1.32^{\mathrm{a}}(1.02-1.61)$ & $1.46^{\mathrm{a}}(1.21-1.70)$ & - \\
\hline CASP10 & - & - & - & - & - \\
\hline CASP8 & $1.84^{\mathrm{a}}(1.22-2.45)$ & $1.87^{\mathrm{b}}(1.01-2.73)$ & - & - & - \\
\hline FASLG & - & - & - & - & - \\
\hline NFKBIA & - & - & $1.31^{\mathrm{a}}(1.06-1.57)$ & $1.50^{\mathrm{a}}(1.21-1.78)$ & $1.47^{\mathrm{a}}(1.15-1.79)$ \\
\hline TNF & - & - & - & - & - \\
\hline TNFRSF1A & - & - & - & $1.42^{\mathrm{a}}(1.02-1.69)$ & - \\
\hline TNFSF10 & - & - & $-1.29^{c}[-1.11-(-1.45)]$ & - & - \\
\hline
\end{tabular}

${ }^{\mathrm{a}} \mathrm{P}<0.050,{ }^{\mathrm{b}} \mathrm{P}<0.059$ and ${ }^{\mathrm{c}} \mathrm{P}=0.038$. CI, confidence interval.

Table IX. Comparison of induced signalling pathways by investigated particles.

\begin{tabular}{|c|c|c|c|c|c|c|c|}
\hline & \multirow{2}{*}{$\begin{array}{c}\begin{array}{c}\text { Direct } \\
\text { genotoxicity }\end{array} \\
\begin{array}{c}\text { DNA damage } \\
\text { and repair }\end{array}\end{array}$} & \multicolumn{3}{|c|}{$\begin{array}{c}\text { Indirect } \\
\text { genotoxicity }\end{array}$} & \multirow{2}{*}{$\begin{array}{c}\begin{array}{c}\text { Initiation and } \\
\text { promotion of } \\
\text { carcinogenesis }\end{array} \\
\begin{array}{c}\text { Proliferation and } \\
\text { carcinogenesis }\end{array}\end{array}$} & \multicolumn{2}{|c|}{$\begin{array}{l}\text { Acute toxicity } \\
\text { and/or } \\
\text { genotoxicity }\end{array}$} \\
\hline & & $\begin{array}{c}\text { Oxidative or } \\
\text { metabolic stress }\end{array}$ & $\begin{array}{l}\text { Growth arrest and } \\
\text { senescence }\end{array}$ & Inflammation & & Heat shock & Apoptosis \\
\hline Crocidolite & XX & XX & $\mathrm{X}$ & XX & $(\mathrm{X})$ & XX & $\mathrm{XX}$ \\
\hline Chrysotile & $X$ & $X$ & $X$ & $\mathrm{X}$ & $(\mathrm{X})$ & $\mathrm{X}$ & $(\mathrm{X})$ \\
\hline $\mathrm{TiO}_{2}$ Anastas & - & $X$ & $(\mathrm{X})$ & $(\mathrm{X})$ & $(\mathrm{X})$ & XX & X \\
\hline $\mathrm{ZrO}_{2}$ & $(\mathrm{X})$ & $X$ & - & - & - & $(\mathrm{X})$ & $\mathrm{XX}$ \\
\hline Hematite & - & $\mathrm{X}$ & - & $X$ & - & XX & $(\mathrm{X})$ \\
\hline
\end{tabular}

$\mathrm{XX}$, high mRNA-induction; $\mathrm{X}$, moderate mRNA-induction; (X), low mRNA-induction; $\mathrm{TiO}_{2}$, titanium dioxide; $\mathrm{ZrO}_{2}$, zirconium dioxide.

'heat shock' pathway, while crocidolite and zirconium dioxide provoked the most changes in mRNA expression of signalling molecules of the 'apoptosis pathway'. Chrysotile showed a moderate increase of 'heat shock' genes and only a moderate increase of 'apoptosis' genes (Tables VII and VIII).

A comparison of the pathways induced by crocidolite, chrysotile, titanium dioxide, zirconium dioxide and hematite is provided in Table IX.

\section{Discussion}

In this study, we compared the ability of two different fibres (crocidolite and chrysotile) and three different sized particles (titanium dioxide, zirconium dioxide and hematite) to induce the mRNA expression of signalling molecules involved in diverse pathways. We characterized the toxicologically relevant chemical and physical properties of the fibres and particles to ensure the comparability of the present results. UICC crocidolite South African and UICC chrysotile 'A' are asbestos fibres, and their cytotoxic and genotoxic potential is well studied. The selected biopersistent dust particles, titanium dioxide (100-200 nm) and zirconium dioxide (50-100 $\mathrm{nm}$ ), were of the same origin as formerly used in vivo (29). After intratracheal installation, both particles induced lung tumours in female SPF Wistar rats (29). Hematite $(20 \mathrm{~nm})$, the smallest of all particles, was investigated, to observe whether the obtained reaction may be provoked by the iron content.

Asbestos fibres caused the most relevant changes in gene expression of all tested pathways. This finding is in accordance with the general knowledge that crocidolite as well as chrysotile are asbestos fibres with a high cytotoxic and genotoxic effect $(2,20,21,30,31)$. A literature search, including in vitro analysis, animal experiments and epidemiological studies, confirmed that all fibre types show comparable harmful effects (32). Chrysotile is, due to its higher solubility, less bio-persistent than the crocidolite (33). Since our study determines the early effects ( $48 \mathrm{~h}$ ) of fibres and particles, the 5-year clearance rate is of minor relevance to our results. In accordance with our study, it appears that chrysotile and crocidolite 
develop their genotoxicity due to direct and indirect (inflammatory driven) molecular mechanisms (18,19,34-36).

The iron content appears to not to be of major relevance for the observed induction of direct genotoxicity or the initiation and promotion of carcinogenesis, since these pathways are not induced by hematite (Fe $70 \%)$ but by zirconium dioxide ( $\mathrm{Fe} 0 \%)$. In a study by Schürkes et al, the iron content appeared not to be relevant for the induction of 8-hydroxydeoxyguanosine $(8-\mathrm{OHdG})$, since fibres with different iron amounts (0.025-20\%) revealed comparable results (35). In the present study, nano-sized hematite and titanium dioxide showed an inflammatory and oxidative stress response and a high increase in gene expression attributed to the 'heat shock' pathway. These findings are in accordance with the results of Park et al, where single intratracheal instillation of iron nanoparticles (NP) in mice elevated the expression of many genes related to inflammation or tissue damage, such as heat shock proteins (37). Additionally, significant generation of ROS was described for titanium dioxide-NP and hematite $(9,24,38)$. None of the investigated genes of the 'DNA damage and repair' pathway were induced by hematite or titanium dioxide in our study. Nanoparticles of hematite but not those of titanium dioxide induced significant DNA-breakage, measured by the Comet-assay in IMR-90 cells. DNA-damage and cytotoxic effects by hematite in BEAS-2B cells were not observed until a concentration of $50 \mu \mathrm{g} / \mathrm{cm}^{2}$ was used (9). On the contrary to ultrafine titanium dioxide, there were no significant alterations in micronuclei induction by fine titanium dioxide observed in Syrian hamster embryo cells (23). Incorporation into human lung cells was described for fine and ultrafine titanium dioxide as well as for hematite $(24,39)$.

Notably, Cyclin D1 which, as a regulatory subunit of CDK4 or CDK6, promotes cell cycle progression through G1-phase is significantly upregulated by titanium dioxide (relative expression 2.36) correspondingly with chrysotile (relative expression 1.89) and crocidolite (relative expression 1.57). The deregulation of cyclin D1 plays an important role in tumorigenesis and has frequently been linked to various types of human cancer (40).

Zirconium dioxide with particle sizes between 50 and $100 \mathrm{~nm}$ induced molecules attributed to the 'oxidative or metabolic stress' pathway, which suggests an indirect genotoxicity. We also found a high increase of apoptotic molecules. Zirconium dioxide induced UNG, which eliminates uracil from DNA molecules by cleaving the N-glycosylic bond and initiates the base-excision repair (BER) pathway. Uracil appearing in DNA, for example as a result of cytosine deamination, is potentially mutagenic and deleterious for cell regulation (41).

In particular, properties such as size, geometry, chemical composition and surface behaviour of particles play important roles in interaction with cells and modify their pathogenicity. Many published studies are missing detailed information on properties and the concentration of the particles used, which makes it difficult to compare results.

Our study and recent reports in the literature demonstrate that gene expression profiling in human lung epithelial cells can be an important tool for analyzing the pathogenicity of potentially harmful fibres and particles (42-44). Gene expression profiling, for example in response to asbestos, is valuable to define early molecular effects as demonstrated in diverse human cells, such as normal human bronchial epithelial cells (NHEC) (45), human lung adenocarcinoma cells (A549) $(46,47)$, SV40-transformed human bronchial epithelial cells (BEAS-2B) and SV40-immortalized pleural mesothelial cells (MET5A) (47). Changes in gene expression are also valuable to determine the pathogenicity pathway of asbestos fibres, as demonstrated in the human mesothelial (LP9/TERT-1) cell line (42).

In further studies, new particles can be screened to complete the toxicological knowledge on the molecular effects and to assess potentially hazardous risks. Altogether, analysis of gene expression profiles may play an important role in the early detection of fibres or potential hazards of particles to human health.

\section{Acknowledgements}

This study was supported by the E.W. Baader-Stiftung supervised by the German Stiftungszentrum, Barkhovenallee 1, 45239 Essen, Germany, Az. T007/20368/2010/sm.

\section{References}

1. DFG: List of MAK and BAT Values 2011. Wiley-VCH Verlag GmbH \& Co. KGaA, Weinheim, 2011.

2. Mossman BT, Bignon J, Corn M, Seaton A and Gee JB: Asbestos: scientific developments and implications for public policy. Science 247: 294-301, 1990.

3. Kamp DW: Asbestos-induced lung diseases: an update. Transl Res 153: 143-152, 2009.

4. Shukla A, MacPherson MB, Hillegass J, Ramos-Nino ME, Alexeeva V, Vacek PM, Bond JP, Pass HI, Steele C and Mossman BT: Alterations in gene expression in human mesothelial cells correlate with mineral pathogenicity. Am J Respir Cell Mol Biol 41: 114-123, 2009.

5. Lemaire I and Ouellet S: Distinctive profile of alveolar macrophage-derived cytokine release induced by fibrogenic and nonfibrogenic mineral dusts. J Toxicol Environ Health 47: 465-478, 1996.

6. Robledo R and Mossman B: Cellular and molecular mechanisms of asbestos-induced fibrosis. J Cell Physiol 180: 158-166, 1999.

7. Mossman BT and Churg A: Mechanisms in the pathogenesis of asbestosis and silicosis. Am J Respir Crit Care Med 157: 1666-1680, 1998.

8. Kamp DW and Weitzman SA: The molecular basis of asbestos induced lung injury. Thorax 54: 638-652, 1999.

9. Bhattacharya K, Davoren M, Boertz J, Schins RP, Hoffmann E and Dopp E: Titanium dioxide nanoparticles induce oxidative stress and DNA-adduct formation but not DNA-breakage in human lung cells. Part Fibre Toxicol 6: 17, 2009.

10. Hext PM, Tomenson JA and Thompson P: Titanium dioxide: inhalation toxicology and epidemiology. Ann Occup Hyg 49: 461-472, 2005

11. Bernard BK, Osheroff MR, Hofmann A and Mennear JH: Toxicology and carcinogenesis studies of dietary titanium dioxide-coated mica in male and female Fischer 344 rats. J Toxicol Environ Health 29: 417-429, 1990.

12. Hart GA and Hesterberg TW: In vitro toxicity of respirable-size particles of diatomaceous earth and crystalline silica compared with asbestos and titanium dioxide. J Occup Environ Med 40: 29-42, 1998.

13. Gurr JR, Wang AS, Chen $\mathrm{CH}$ and Jan KY: Ultrafine titanium dioxide particles in the absence of photoactivation can induce oxidative damage to human bronchial epithelial cells. Toxicology 213: 66-73, 2005.

14. Kang SJ, Kim BM, Lee YJ and Chung HW: Titanium dioxide nanoparticles trigger p53-mediated damage response in peripheral blood lymphocytes. Environ Mol Mutagen 49: 399-405, 2008.

15. DaNa: Zirconium dioxide. http://nanopartikel.info/cms/lang/en/ Wissensbasis/Zirkoniumdioxid. Accessed October 8, 2013. 
16. NanoCare Project Partners: NanoCare. Health Related Aspects of Nanomaterials. Final Scientific Report. T.A.J. Kuhlbusch, H.F. Krug and K. Nau (eds). 1st edition. DECHEMA e.V. (in cooperation with the NanoCare Project Consortium), Frankfurt am Main, 2009.

17. Schneider J, Walter D, Brückel B and Rödelsperger K: Primary particles and their agglomerate formation as modifying risk factors of nonfibrous nanosized dust. J Toxicol Environ Health A 76: 131-141, 2013.

18. Dopp E, Schuler M, Schiffmann D and Eastmond DA: Induction of micronuclei, hyperdiploidy and chromosomal breakage affecting the centric/pericentric regions of chromosomes 1 and 9 in human amniotic fluid cells after treatment with asbestos and ceramic fibers. Mutat Res 377: 77-87, 1997.

19. Burmeister B, Schwerdtle T, Poser I, Hoffmann E, Hartwig A, Müller WU, Rettenmeier AW, Seemayer NH and Dopp E: Effects of asbestos on initiation of DNA damage, induction of DNA-strand breaks, P53-expression and apoptosis in primary, SV40-transformed and malignant human mesothelial cells. Mutat Res 558: 81-92, 2004.

20. Dopp E, Yadav S,AnsariFA, Bhattacharya K, von RecklinghausenU, Rauen U, Rödelsperger K, Shokouhi B, Geh S and Rahman Q: ROS-mediated genotoxicity of asbestos-cement in mammalian lung cells in vitro. Part Fibre Toxicol 2: 9, 2005.

21. Poser I, Rahman Q, Lohani M, Yadav S, Becker HH, Weiss DG, Schiffmann D and Dopp E: Modulation of genotoxic effects in asbestos-exposed primary human mesothelial cells by radical scavengers, metal chelators and a glutathione precursor. Mutat Res 559: 19-27, 2004.

22. Bhattacharya $\mathrm{K}$ : Comparative analysis of fine and nanoparticles for cellular uptake, oxidative stress and genomic damage in human lung cells (unpublished $\mathrm{PhD}$ thesis). University of Duisburg-Essen, 2009.

23. Rahman Q, Lohani M, Dopp E, Pemsel H, Jonas L, Weiss DG and Schiffmann D: Evidence that ultrafine titanium dioxide induces micronuclei and apoptosis in Syrian hamster embryo fibroblasts. Environ Health Perspect 110: 797-800, 2002.

24. Bhattacharya K, Hoffmann E, Schins RF, et al: Comparison of micro- and nanoscale $\mathrm{Fe}^{+3}$-containing (Hematite) particles for their toxicological properties in human lung cells in vitro. Toxicol Sci 126: 173-182, 2012.

25. Pfaffl MW: A new mathematical model for relative quantification in real-time RT-PCR. Nucleic Acids Res 29: e45, 2001.

26. Buxbaum G and Paff G (eds): Industrial Inorganic Pigments. 3rd edition. Wiley-VCH Verlag GmbH \& Co. KGaA, Weinheim, 2005.

27. Buxbaum G and Printzen H: Ullmann's Encyclopedia of Industrial Chemistry. Vol A20. 5th edition. VCH Verlagsgesellschaft $\mathrm{mbH}$, Weinheim, p297, 1992.

28. Walter D: Characterization of synthetic hydrous hematite pigments. Thermochimica Acta 445: 195-199, 2006.

29. Pott F and Roller M: Carcinogenicity study with nineteen granular dusts in rats. Eur J Oncol 10: 249-281, 2005.

30. Jones JSP, Smith PG, Pooley FD, et al: Biological effects of mineral fibres. In: IARC Monographs on the Evaluation of Carcinogenic Risks to Humans. WHO International Agency for Research on Cancer, Lyon, pp637-653, 1980.

31. WHO, IARC: IARC Monographs on the Evaluation of Carcinogenic Risks to Humans Overall Evaluations of Carcinogenicity: An Updating of IARC Monographs Volumes 1 to 42. Supplement 7. IARC, Lyon, 1987. http://monographs.iarc.fr/ ENG/Monographs/suppl7/suppl7.pdf. Accessed October 8, 2013.

32. Baur X, Schneider J, Woitowitz HJ and Velasco Garrido M: Do advers health effects of chrysotile and amphibole asbestos differ? Pneumologie 66: 497-506, 2012 (In German).
33. Bernstein DM, Rogers R and Smith P: The biopersistence of Canadian chrysotile asbestos following inhalation. Inhal Toxicol 15: 1247-1274, 2003.

34. Dopp E, Poser I and Papp T: Interphase fish analysis of cell cycle genes in asbestos-treated human mesothelial cells (HMC), SV40transformed HMC (MeT-5A) and mesothelioma cells (COLO). Cell Mol Biol (Noisy-le-grand) 48: OL271-OL277, 2002.

35. Schürkes C, Brock W, Abel J and Unfried K: Induction of 8-hydroxydeoxyguanosine by man made vitreous fibres and crocidolite asbestos administered intraperitoneally in rats. Mutat Res 553: 59-65, 2004

36. Ruosaari S, Hienonen-Kempas T, Puustinen A, Sarhadi VK, Hollmén J, Knuutila S, Saharinen J, Wikman H and Anttila S: Pathways affected by asbestos exposure in normal and tumour tissue of lung cancer patients. BMC Med Genomics 1: 55, 2008.

37. Park EJ, Kim H, Kim Y, Yi J, Choi K and Park K: Inflammatory responses may be induced by a single intratracheal instillation of iron nanoparticles in mice. Toxicology 275: 65-71, 2010.

38. Könczöl M, Ebeling S, Goldenberg E, Treude F, Gminski R, Gieré R, Grobéty B, Rothen-Rutishauser B, Merfort I and Mersch-Sundermann V: Cytotoxicity and genotoxicity of size-fractionated iron oxide (magnetite) in A549 human lung epithelial cells: role of ROS, JNK, and NF-kappaB. Chem Res Toxicol 24: 1460-1475, 2011.

39. Singh S, Shi T, Duffin R, Albrecht C, van Berlo D, Höhr D, Fubini B, Martra G, Fenoglio I, Borm PJ and Schins RP: Endocytosis, oxidative stress and IL-8 expression in human lung epithelial cells upon treatment with fine and ultrafine $\mathrm{TiO}_{2}$ : role of the specific surface area and of surface methylation of the particles. Toxicol Appl Pharmacol 222: 141-151, 2007.

40. Witzel, II, Koh LF and Perkins ND: Regulation of cyclin D1 gene expression. Biochem Soc Trans 38: 217-222, 2010.

41. Zharkov DO, Mechetin GV and Nevinsky GA: Uracil-DNA glycosylase: structural, thermodynamic and kinetic aspects of lesion search and recognition. Mutat Res 685: 11-20, 2010

42. Hillegass JM, Shukla A, MacPherson MB, Bond JP, Steele C and Mossman BT: Utilization of gene profiling and proteomics to determine mineral pathogenicity in a human mesothelial cell line (LP9/TERT-1). J Toxicol Environ Health A 73: 423-436, 2010.

43. Perkins TN, Shukla A, Peeters PM, Steinbacher JL, Landry CC, Lathrop SA, Steele C, Reynaert NL, Wouters EF and Mossman BT: Differences in gene expression and cytokine production by crystalline vs. amorphous silica in human lung epithelial cells. Part Fibre Toxicol 9: 6, 2012.

44. Huang YC, Karoly ED, Dailey LA, Schmitt MT, Silbajoris R, Graff DW and Devlin RB: Comparison of gene expression profiles induced by coarse, fine, and ultrafine particulate matter. J Toxicol Environ Health A 74: 296-312, 2011.

45. Belitskaya-Levy I, Hajjou M, Su WC, Yie TA, Tchou-Wong KM, Tang MS, Goldberg JD and Rom WN: Gene profiling of normal human bronchial epithelial cells in response to asbestos and benzo(a)pyrene diol epoxide (BPDE). J Environ Pathol Toxicol Oncol 26: 281-294, 2007

46. Hevel JM, Olson-Buelow LC, Ganesan B, Stevens JR, Hardman JP and Aust AE: Novel functional view of the crocidolite asbestos-treated A549 human lung epithelial transcriptome reveals an intricate network of pathways with opposing functions. BMC Genomics 9: 376, 2008

47. Nymark P, Lindholm PM, Korpela MV, Lahti L, Ruosaari S, Kaski S, Hollmén J, Anttila S, Kinnula VL and Knuutila S: Gene expression profiles in asbestos-exposed epithelial and mesothelial lung cell lines. BMC Genomics 8: 62, 2007. 